\title{
REES ALGEBRAS AND MIXED MULTIPLICITIES
}

\author{
J. K. VERMA
}

(Communicated by Louis J. Ratliff, Jr.)

To Professor David Rees, in honor of his seventieth birthday

\begin{abstract}
Let $(R, m)$ be a local ring of positive dimension $d$ and $I$ and $J$ two $m$-primary ideals of $R$. Let $T$ denote the Rees algebra $R[J t]$ localized at the maximal homogeneous ideal $(m, J t)$. It is proved that

$$
e((I, J t) T)=e_{0}(I \mid J)+e_{1}(I \mid J)+\cdots+e_{d-1}(I \mid J),
$$

where $e_{i}(I \mid J), i=0,1, \ldots, d-1$ are the first $d$ mixed multiplicities of $I$ and $J$. A formula due to Huneke and Sally concerning the multiplicity of the Rees algebra (of a complete zero-dimensional ideal of a two dimensional regular local ring) at its maximal homogeneous ideal is recovered.
\end{abstract}

1. Introduction. Bhattacharya [B] and Risler and Teissier [T] obtained a useful and natural generalization of the Hilbert function and multiplicity of an ideal. To elaborate, let $I$ and $J$ be $m$-primary ideals of a local ring $(R, m)$ of positive dimension $d$. Let $l$ denote length and $\mathbf{N}$ denote the set of positive integers. Then the function $B(r, s): \mathrm{N}^{2} \rightarrow \mathrm{N}$ defined as $B(r, s)=l\left(R / I^{r} J^{s}\right)$ is a polynomial of total degree $d$ in $r$ and $s$ for large values of $r$ and $s$. This polynomial is called the Bhattacharya polynomial of $I$ and $J$. The terms of total degree $d$ in the Bhattacharya polynomial of $I$ and $J$ have the form

$$
\frac{1}{d !}\left\{e_{0}(I \mid J) r^{d}+\cdots+\left(\begin{array}{c}
d \\
i
\end{array}\right) e_{i}(I \mid J) r^{d-i} s^{i}+\cdots+e_{d}(I \mid J) s^{d}\right\} .
$$

Here $e_{0}(I \mid J), \ldots, e_{d}(I \mid J)$ are all positive integers, called the mixed multiplicities of $I$ and $J$. Rees [R1] observed that $e_{0}(I \mid J)=e(I)$ and $e_{d}(I \mid J)=e(J)$ where $e$ denotes multplicity. A fundamental contribution of Risler and Teissier is to identify $e_{i}(I \mid J)$ as the multiplicity of an ideal generated by $d-i$ elements from $I$ and $i$ elements from $J$ chosen "sufficiently generally." Rees has reinterpreted the phrase "sufficiently generally" by introducing the concepts of joint reductions [R4] and independent set of general elements of a collection of ideals [R3].

Let $R[J t]$ denote the Rees algebra of the ideal $J, N$ denote its maximal homogeneous ideal $(m, J t)$ and set $T=R[J t]_{N}$. The purpose of this paper is to prove that

In particular,

$$
e((I, J t) T)=e_{0}(I \mid J)+\cdots+e_{d-1}(I \mid J) .
$$

$$
e(T)=e_{0}(m \mid J)+\cdots+e_{d-1}(M \mid J) .
$$

Received by the editors January 4, 1988 and, in revised form, February 8, 1988. Presented at the 90th Summer meeting of the AMS, August 6, 1987.

1980 Mathematics Subject Classification (1985 Revision). Primary 13A15; Secondary 13H15.

Key words and phrases. Bhattacharya polynomial, contracted ideal, integral closure of an ideal, joint reduction, mixed multiplicities, Rees algebra, regular local ring. 
In $\S 2$, we recall the necessary definitions and results. $\S 3$ is devoted to the proof of the main theorem. In $\S 4$ we specialize to two-dimensional regular local rings and recover a formula due to Huneke and Sally [HS] which originally inspired our main theorem. Finally we present an example in $\S 5$ which illustrates our main theorem.

\section{Preliminaries.}

(2.1) By a result due to Valla $[\mathbf{V}], \operatorname{dim} R[J t]=d+1$ provided $d>0$.

(2.2) It follows from the Bhattacharya polynomial of $I$ and $J$ that for all positive integers $r$ and $s$,

$$
e\left(I^{r} J^{s}\right)=r^{d} e(I)+\cdots+\left(\begin{array}{c}
d \\
i
\end{array}\right) e_{i}(I \mid J) r^{d-i} s^{i}+\cdots+s^{d} e(J) .
$$

In particular, by putting $r=1=s$ we get

$$
e(I J)=e(I)+\cdots+\left(\begin{array}{l}
d \\
i
\end{array}\right) e_{i}(I \mid J)+\cdots+e(J) .
$$

If $J=I$ then for all positive integers $r$ and $s$

$$
e\left(I^{r+s}\right)=r^{d} e(I)+\cdots+\left(\begin{array}{c}
d \\
i
\end{array}\right) e_{i}(I \mid I) r^{d-i} s^{i}+\cdots+s^{d} e(I)
$$

Therefore, it follows that $e_{i}(I \mid I)=e(I)$ for all $i$.

(2.3) Recall that an ideal $K \subset I$ is called a reduction of $I$ if there is a positive integer $n$ such that $K I^{n}=I^{n+1}[\mathbf{N R}]$. It easily follows that $e(K)=e(I)$. Moreover, if $L$ is a reduction of $J$ then $K^{r} L^{s}$ is a reduction of $I^{r} J^{s}$ for all positive integers $r$ and $s$. Thus

$$
e\left(K^{r} L^{s}\right)=\sum_{i=0}^{d}\left(\begin{array}{l}
d \\
i
\end{array}\right) e_{i}(K \mid L) r^{d-i} s^{i}
$$

and

$$
e\left(I^{r} J^{s}\right)=\sum_{i=0}^{d}\left(\begin{array}{l}
d \\
i
\end{array}\right) e_{i}(I \mid J) r^{d-i} s^{i}
$$

Since $e\left(K^{r} L^{s}\right)=e\left(I^{r} J^{s}\right)$, we conclude that $e_{i}(I \mid J)=e_{i}(K \mid L)$.

(2.4) Let $m, n$ be positive integers. Then for all positive integers $r$ and $s$,

$$
e\left(I^{r m} J^{s n}\right)=\sum_{i=0}^{d}\left(\begin{array}{l}
d \\
i
\end{array}\right) e_{i}(I \mid J)(r m)^{d-i}(s n)^{i} .
$$

We also have

$$
e\left(I^{r m} J^{s n}\right)=\sum_{i=0}^{d}\left(\begin{array}{l}
d \\
i
\end{array}\right) e_{i}\left(I^{m} \mid J^{n}\right) r^{d-i} s^{i} .
$$

Comparing the above two expressions we get

$$
e_{i}\left(I^{m} \mid J^{n}\right)=m^{d-i} n^{i} e_{i}(I \mid J) .
$$

(2.5) An element $x \in R$ is said to be integral over an ideal $I$ of $R$ if $x$ satisfies an equation of the form

$$
x^{n}+a_{1} x^{n-1}+\cdots+a_{n}=0 \quad \text { where } a_{i} \in I^{i} \quad \text { for } i=1, \ldots, n .
$$


Equivalently, $x$ is integral over $I$ if and only if for some $n, I(I, x)^{n-1}=(I, x)^{n}$. In other words, $I$ is a reduction of $(I, x)$. It follows from this fact that an ideal $I \subset J$ is a reduction of an ideal $J$ if and only if every element of $J$ is integral over $I$. Elements of $R$ which are integral over $I$ form an ideal which we denote by $I_{a}$. An ideal $I$ is called a complete ideal if $I=I_{a}$.

(2.6) LEMMA. Let $m$ and $n$ be positive integers with $m \leq n$. Let $\delta_{m n}$ denote the Kronecker $\delta$-function. Then

$$
\sum_{k=m}^{n}\left(\begin{array}{l}
n \\
k
\end{array}\right)\left(\begin{array}{l}
k \\
m
\end{array}\right)(-1)^{k}=(-1)^{m} \delta_{m n} .
$$

PROOF. If $m=n$ then both sides are clearly equal. Suppose $m<n$. Then

$$
\begin{aligned}
\sum_{k=m}^{n}\left(\begin{array}{l}
n \\
k
\end{array}\right)\left(\begin{array}{c}
k \\
m
\end{array}\right)(-1)^{k} & =\sum_{k=m}^{n} \frac{n !}{(n-k) ! k !} \cdot \frac{k !}{(k-m) ! m !}(-1)^{k} \\
& =\frac{n !}{(n-m) ! m !} \sum_{k=m}^{n} \frac{(n-m) !(-1)^{k}}{(n-k) !(k-m) !} \\
& =\left(\begin{array}{c}
n \\
m
\end{array}\right) \sum_{j=0}^{n-m} \frac{(n-m) !(-1)^{j+m}}{(n-j-m) ! j !} \\
& =(-1)^{m}\left(\begin{array}{c}
n \\
m
\end{array}\right) \sum_{j=0}^{n-m}\left(\begin{array}{c}
n-m \\
j
\end{array}\right)(-1)^{j} \\
& =(-1)^{m}\left(\begin{array}{c}
n \\
m
\end{array}\right)(1-1)^{n-m}=0 .
\end{aligned}
$$

(2.7) Lemma. For $j=0,1,2, \ldots, d$

$$
\sum_{p=0}^{d-j}\left(\begin{array}{l}
d \\
j
\end{array}\right)\left(\begin{array}{c}
d-j \\
p
\end{array}\right) \frac{(-1)^{p}(d+1)}{j+p+1}=1 .
$$

PROOF.

$$
\begin{aligned}
\sum_{p=0}^{d-j}\left(\begin{array}{l}
d \\
j
\end{array}\right)\left(\begin{array}{c}
d-j \\
p
\end{array}\right) \frac{(-1)^{p}(d+1)}{j+p+1} & =\sum_{p=j}^{d}\left(\begin{array}{l}
d \\
j
\end{array}\right)\left(\begin{array}{c}
d-j \\
p-j
\end{array}\right) \frac{(d+1)(-1)^{p-j}}{p+1} \\
& =\sum_{p=j}^{d} \frac{d !(d-j) ! p !(d+1)(-1)^{p-j}}{(d-j) ! j !(d-p) !(p-j) ! p !(p+1)} \\
& =\sum_{p=j}^{d}\left(\begin{array}{l}
d+1 \\
p+1
\end{array}\right)\left(\begin{array}{l}
p \\
j
\end{array}\right)(-1)^{p-j} \\
& =\sum_{p=0}^{d}\left(\begin{array}{l}
d+1 \\
p+1
\end{array}\right)\left(\begin{array}{l}
p \\
j
\end{array}\right)(-1)^{p-j} .
\end{aligned}
$$

For $j=0$ the above sum reduces to

$$
\sum_{p=0}^{d}\left(\begin{array}{l}
d+1 \\
p+1
\end{array}\right)(-1)^{p}=1-(1-1)^{d+1}=1
$$


Suppose that we have proved that the equality is true for $j=0,1, \ldots, q-1$. Then we show it for $j=q$

$$
\begin{aligned}
& \sum_{p=0}^{d}\left(\begin{array}{l}
d+1 \\
p+1
\end{array}\right)\left(\begin{array}{l}
p \\
q
\end{array}\right)(-1)^{p-q} \\
& \quad=\sum_{p=0}^{d}\left(\begin{array}{l}
d+1 \\
p+1
\end{array}\right)\left(\begin{array}{c}
p+1 \\
q
\end{array}\right)(-1)^{p-q}+\sum_{p=0}^{d}\left(\begin{array}{c}
d+1 \\
p+1
\end{array}\right)\left(\begin{array}{c}
p \\
q-1
\end{array}\right)(-1)^{p-(q-1)} .
\end{aligned}
$$

The first sum in the above equation is zero by Lemma 2.6 and the second sum is equal to 1 by the induction hypothesis.

(2.8) LEMMA. For positive integers $n$ and $r$,

$$
1^{r}+2^{r}+\cdots+n^{r}=\frac{n^{r+1}}{r+1}+\frac{1}{2} n^{r}+\cdots .
$$

PROOF (R. SivARAMAKRISHNAN). Write $S_{r}(n)=1^{r}+2^{r}+\cdots+n^{r}$. By $[\mathbf{R i}$, p. 159] we have the identity

$$
S_{r}(n)-\sum_{k=0}^{r}\left(\begin{array}{l}
r \\
k
\end{array}\right)(-1)^{r-k} S_{k}(n)=n^{r}
$$

Change $r$ to $r+1$ to obtain

$$
S_{r+1}(n)-\left\{S_{r+1}(n)-\left(\begin{array}{c}
r+1 \\
r
\end{array}\right) S_{r}(n)+\left(\begin{array}{c}
r+1 \\
r-1
\end{array}\right) S_{r-1}(n)+\cdots\right\}=n^{r+1}
$$

Hence

$$
\left(\begin{array}{c}
r+1 \\
1
\end{array}\right) S_{r}(n)-\left(\begin{array}{c}
r+1 \\
2
\end{array}\right) S_{r-1}(n)+\cdots=n^{r+1}
$$

The lemma is trivial for $r=1$. Assume that it is proved for all positive integers less than or equal to $r-1$. By the induction hypothesis we get

$$
(r+1) S_{r}(n)-\left(\begin{array}{c}
r+1 \\
2
\end{array}\right)\left[\frac{n^{r}}{r}+\frac{1}{2} n^{r-1}+\cdots\right]+\cdots=n^{r+1}
$$

Thus

$$
S_{r}(n)=\frac{n^{r+1}}{r+1}+\frac{1}{2} n^{r}+\cdots
$$

REMARK. For further discussion of $S_{r}(n)$, see [Ra, Chapter 1] where $S_{r}(n)$ is expressed in terms of Bernoulli polynomials.

\section{The main theorem.}

(3.1) THEOREM. Let $(R, m)$ be a local ring of positive dimensiond and $I, J \mathrm{~m}$ primary ideals of $R$. Let $N$ denote the unique maximal homogeneous ideal $(m, J t)$ of the Rees algebra $R[J t]$ and set $T=R[J t]_{N}$. Then

$$
e((I, J t) T)=e_{0}(I \mid J)+\cdots+e_{d-1}(I \mid J) .
$$

Proof. Let $K$ denote the ideal $(I, J t) T$. To compute $e(K)$ we compute $l\left(T / K^{n}\right)$ for large $n$. The direct sum decompositions of $R[J t]$ and $(I, J t)^{n}$ are

$$
\begin{gathered}
R[J t]=R \oplus J t \oplus J^{2} t^{2} \oplus \cdots \oplus J^{n-1} t^{n-1} \oplus J^{n} t^{n} \oplus \cdots ; \\
(I, J t)^{n}=I^{n} \oplus I^{n-1} J t \oplus I^{n-2} J^{2} t^{2} \oplus \cdots \oplus I J^{n-1} t^{n-1} \oplus J^{n} t^{n} \oplus \cdots
\end{gathered}
$$


Therefore,

$$
\begin{aligned}
l\left(T / K^{n}\right) & =\sum_{i=0}^{n} l\left(J^{i} / I^{n-i} J^{i}\right) \\
& =\sum_{i=0}^{n} l\left(R / I^{n-i} J^{i}\right)-\sum_{i=0}^{n} l\left(R / J^{i}\right) .
\end{aligned}
$$

Now we make use of the Bhattacharya polynomial of $I$ and $J$ to compute $l\left(R / I^{n-i} J^{i}\right)$. We may assume that the Bhattacharya function of $I$ and $J$ coincides with the Bhattacharya polynomial of $I$ and $J$, since this assumption does not affect the leading coefficient of the Hilbert polynomial of $K$. Therefore we obtain, by setting $e_{k}=e_{k}(I \mid J)$ for $k=0,1, \ldots, d$,

$$
\begin{aligned}
\sum_{i=0}^{n} l\left(R / I^{n-i} J^{i}\right) & =\sum_{i=0}^{n} \sum_{k=0}^{d}\left(\begin{array}{l}
d \\
k
\end{array}\right)\left(e_{k} / d !\right)(n-i)^{d-k} \cdot i^{k}+\cdots \\
& =\sum_{i=0}^{n} \sum_{k=0}^{d}\left(\begin{array}{l}
d \\
k
\end{array}\right)\left(\left(e_{k}\right) \cdot\left(i^{k}\right) / d !\right) \sum_{r=0}^{d-k}\left(\begin{array}{c}
d-k \\
r
\end{array}\right) n^{d-k-r} i^{r}(-1)^{r}+\cdots \\
& =\sum_{k=0}^{d} \sum_{r=0}^{d-k}\left(\begin{array}{l}
d \\
k
\end{array}\right)\left(\begin{array}{c}
d-k \\
r
\end{array}\right)\left(e_{k} / d !\right) n^{d-k-r}(-1)^{r} \sum_{i=0}^{n} i^{k+r}+\cdots \\
& =\sum_{k=0}^{d} \sum_{r=0}^{d-k}\left(\begin{array}{l}
d \\
k
\end{array}\right)\left(\begin{array}{c}
d-k \\
r
\end{array}\right)\left(e_{k} / d !\right)(-1)^{r} \frac{n^{d+1}}{k+r+1}+\cdots \\
& =\frac{n^{d+1}}{(d+1) !} \sum_{k=0}^{d}\left\{\sum_{r=0}^{d-k}\left(\begin{array}{l}
d \\
k
\end{array}\right)\left(\begin{array}{c}
d-k \\
r
\end{array}\right) \frac{(-1)^{r}(d+1)}{k+r+1}\right\} e_{k}+\cdots \\
& =\frac{n^{d+1}}{(d+1) !}\left(e_{0}+e_{1}+\cdots+e_{d}\right)+\cdots
\end{aligned}
$$

Therefore for large $n$

$$
\begin{aligned}
l\left(T / K^{n}\right) & =\frac{n^{d+1}}{(d+1) !}\left(e_{0}+e_{1}+\cdots+e_{d}\right)-\sum_{i=0}^{n} \frac{i^{d}}{d !} e(J)+\cdots \\
& =\frac{n^{d+1}}{(d+1) !}\left(e_{0}+e_{1}+\cdots+e_{d}\right)-\left(\frac{n^{d+1}}{(d+1) !} e(J)\right)+\cdots \\
& =\frac{n^{d+1}}{(d+1) !}\left(e_{0}+e_{1}+\cdots+e_{d-1}\right)+\cdots
\end{aligned}
$$

Hence $e(K)=e_{0}+e_{1}+\cdots+e_{d-1}$ and the proof is complete.

(3.2) COROLlARY. Let $(R, m)$ be a local ring of positive dimension $d$. Let $r$ be a positive integer. Then by setting $N=\left(m, m^{r} t\right)$ we have

$$
e\left(R\left[m^{r} t\right]_{N}\right)=e(R)\left(1+r+\cdots+r^{d-1}\right) .
$$

PROOF. By the theorem

$$
\begin{aligned}
e\left(R\left[m^{r} t\right]_{N}\right) & =e_{0}\left(m \mid m^{r}\right)+e_{1}\left(m \mid m^{r}\right)+\cdots+e_{d-1}\left(m \mid m^{r}\right) \\
& =e(R)+r e_{1}(m \mid m)+\cdots+r^{d-1} e_{d-1}(m \mid m) \\
& =e(R)\left(1+r+\cdots+r^{d-1}\right) .
\end{aligned}
$$


4. The case of two-dimensional regular local rings. Throughout this section $(R, m)$ will denote a two-dimensional regular local ring. Let $o$ denote the valuation of the quotient field of $R$ such that for $0 \neq x \in R$

$$
o(x)=\max \left\{n \mid x \in m^{n}\right\} .
$$

For an ideal $I$ of $R$, put

$$
o(I)=\min \{o(x) \mid 0 \neq x \in I\} .
$$

Let $N$ denote the unique maximal homogeneous ideal of the Rees algebra $R[I t]$. Huneke and Sally [HS] proved that if $I$ is an $m$-primary complete ideal, then

$$
e\left(R[I t]_{N}\right)=1+o(I) .
$$

This formula originally inspired our main theorem. We recover it as a consequence. First we need to recall some results concerning complete and contracted ideals in a two-dimensional regular local ring. An ideal $I$ of $R$ is called a contracted ideal [ZS, Appendix 5] if there is an $x \in m \backslash m^{2}$ such that $I R[m / x] \cap R=I$. Zariski [ZS, Appendix 5] proved that a complete $m$-primary ideal is contracted, a product of contracted ideals is contracted, and a product of complete ideals is complete. The theory of complete ideals in two-dimensional regular local rings was founded by Zariski in [Z].

Lipman [L] and Rees [R2] proved that if $I$ is an $m$-primary contracted ideal of $R$ then $\mu(I)=$ minimum number of generators for $I=1+o(I)$. Huneke $[\mathbf{H}]$ proved that the converse is true provided $R / m$ is infinite. Now we are ready to prove

(4.1) THEOREM (HUNEKE-SALLY). Let $(R, m)$ be a two-dimensional regular local ring. For an m-primary ideal $I$ of $R$, let $N$ denote the unique maximal homogeneous ideal $(m, I t)$ of the Rees algebra $R[I t]$. Then $e\left(R[I t]_{N}\right)=\mu\left(I_{a}\right)=1+o(I)$.

PROOF. By the main theorem

$$
e\left(R[I t]_{N}\right)=e_{0}(m \mid I)+e_{1}(m \mid I)=1+e_{1}(m \mid I) .
$$

Since $m^{n}$ is complete for all $n, o(I)=o\left(I_{a}\right)$. Also, $e_{1}(m \mid I)=e_{1}\left(m \mid I_{a}\right)$. Therefore we may assume that $I$ is a complete ideal. Hence $I$ is contracted and consequently $I^{r} m^{s}$ is a contracted ideal for all positive integers $r$ and $s$. Therefore $\mu\left(I^{r} m^{s}\right)=$ $s+r o(I)+1$.

Now we calculate the Bhattacharya polynomial of $I$ and $m$. For all $r$ and $s$,

$$
\begin{aligned}
l\left(R / I^{r} m^{s}\right) & =l\left(R / I^{r}\right)+\sum_{i=0}^{s-1} l\left(I^{r} m^{i} / I^{r} m^{i+1}\right)=l\left(R / I^{r}\right)+\sum_{i=0}^{s-1} \mu\left(I^{r} m^{i}\right) \\
& =l\left(R / I^{r}\right)+\sum_{i=0}^{s-1}(i+r o(I)+1)=l\left(R / I^{r}\right)+r s o(I)+s(s+1) / 2 \\
& =l\left(R / I^{r}\right)+r s o(I)+l\left(R / m^{s}\right)=\frac{1}{2}\left\{e(I) r^{2}+2 r s o(I)+s^{2} e(m)\right\}+\cdots
\end{aligned}
$$

(by [H, Theorem 7.2]). Therefore $e_{1}(m \mid I)=o(I)$ which finishes the proof.

5. An example. Let $(R, m)$ be a three-dimensional regular local ring with $(x, y, z)$ a regular system of parameters, i.e., $m=(x, y, z)$. Let $J$ and $I$ denote 
the ideals $\left(x, y^{2}, y z, z^{2}\right)$ and $m J$ respectively. We show that $e\left(R[J t]_{N}\right)=4$ where $N=(m, J t)$. Set $e_{i}(m \mid J)=e_{i}$ for $i=0,1,2,3$. By the main theorem

$$
e\left(R[J t]_{N}\right)=e_{0}+e_{1}+e_{2}=1+e_{1}+e_{2} .
$$

On the other hand,

$$
\begin{aligned}
e(m J) & =e_{0}(m \mid J)+\left(\begin{array}{l}
3 \\
1
\end{array}\right) e_{1}(m \mid J)+\left(\begin{array}{l}
3 \\
2
\end{array}\right) e_{2}(m \mid J)+e_{3}(m \mid J) \\
& =1+3\left(e_{1}+e_{2}\right)+e(J) .
\end{aligned}
$$

Since $y z$ is integral over $J, e(J)=e\left(\left(x, y^{2}, z^{2}\right)\right)$. Since $R$ is Cohen-Macaulay

$$
e\left(\left(x, y^{2}, z^{2}\right)\right)=l\left(R /\left(x, y^{2}, z^{2}\right)\right)=4 \text {. }
$$

Therefore, $e(m J)=5+3\left(e_{1}+e_{2}\right)$. We now calculate the mulitplicity of $I=m J$ by calculating its Hilbert polynomial. For all $n$,

$$
\begin{aligned}
I^{n}= & \left(x^{2 n}, \ldots, x^{2 n-i}(y, z)^{i}, \ldots, x^{n}(y, z)^{n},\right. \\
& \left.x^{n-1}(y, z)^{n+2}, \ldots, x^{n-i}(y, z)^{n+2 i}, \ldots,(y, z)^{3 n}\right) .
\end{aligned}
$$

Therefore, $l\left(R / I^{n}\right)=$ the number of monomials of the form $x^{i}\left(y^{j} z^{k}\right)$ for $i=0,1, \ldots$, $2 n-1$ not belonging to $I^{n}$. An easy counting argument shows that

$$
l\left(R / I^{n}\right)=\sum_{i=1}^{n}\left(\begin{array}{c}
i+1 \\
2
\end{array}\right)+\sum_{i=1}^{n}\left(\begin{array}{c}
n+2 i+1 \\
2
\end{array}\right)
$$

From this we obtain

$$
\begin{gathered}
l(R / I)=\left(\begin{array}{l}
2 \\
2
\end{array}\right)+\left(\begin{array}{l}
4 \\
2
\end{array}\right)=7, \quad l\left(R / I^{2}\right)=\left(\begin{array}{l}
2 \\
2
\end{array}\right)+\left(\begin{array}{l}
3 \\
2
\end{array}\right)+\left(\begin{array}{l}
5 \\
2
\end{array}\right)+\left(\begin{array}{l}
7 \\
2
\end{array}\right)=35 \\
l\left(R / I^{3}\right)=\left(\begin{array}{l}
2 \\
2
\end{array}\right)+\left(\begin{array}{l}
3 \\
2
\end{array}\right)+\left(\begin{array}{l}
4 \\
2
\end{array}\right)+\left(\begin{array}{l}
6 \\
2
\end{array}\right)+\left(\begin{array}{l}
8 \\
2
\end{array}\right)+\left(\begin{array}{c}
10 \\
2
\end{array}\right)=98 \\
l\left(R / I^{4}\right)=\left(\begin{array}{l}
2 \\
2
\end{array}\right)+\left(\begin{array}{l}
3 \\
2
\end{array}\right)+\left(\begin{array}{l}
4 \\
2
\end{array}\right)+\left(\begin{array}{l}
5 \\
2
\end{array}\right)+\left(\begin{array}{l}
7 \\
2
\end{array}\right)+\left(\begin{array}{l}
9 \\
2
\end{array}\right)+\left(\begin{array}{c}
11 \\
2
\end{array}\right)+\left(\begin{array}{c}
13 \\
2
\end{array}\right)=210 .
\end{gathered}
$$

The above expression for $l\left(R / I^{n}\right)$ shows that the Hilbert polynomial of $I$ coincides with the Hilbert function of $I$. Write the Hilbert polynomial in the standard form

$$
l\left(R / I^{n}\right)=f_{0}\left(\begin{array}{c}
n+2 \\
3
\end{array}\right)-f_{1}\left(\begin{array}{c}
n+1 \\
2
\end{array}\right)+f_{2}\left(\begin{array}{l}
n \\
1
\end{array}\right)-f_{3} .
$$

Substitute $n=1,2,3,4$ to obtain the following system of linear equations:

$$
\begin{aligned}
f_{0}-f_{1}+f_{2}-f_{3} & =7 \\
4 f_{0}-3 f_{1}+2 f_{2}-f_{3} & =35 \\
10 f_{0}-6 f_{1}+3 f_{2}-f_{3} & =98 \\
20 f_{0}-10 f_{1}+4 f_{2}-f_{3} & =210 .
\end{aligned}
$$

It turns out that $f_{0}=14, f_{1}=7$, and $f_{2}=f_{3}=0$. Thus the Hilbert polynomial of $I$ is given by

$$
l\left(R / I^{n+1}\right)=14\left(\begin{array}{c}
n+3 \\
3
\end{array}\right)-7\left(\begin{array}{c}
n+2 \\
2
\end{array}\right) \text { for all } n=0,1,2, \ldots
$$


Therefore $e(m J)=14=1+3\left(e_{1}+e_{2}\right)+4$. This gives that $e_{1}+e_{2}=3$ and consequently $e\left(R[J t]_{N}\right)=e_{0}+e_{1}+e_{2}=4$.

Alternatively, we can calculate $e_{1}$ and $e_{2}$ by using joint reductions. Rees [R4] defines these as follows: Let $(R, m)$ be a $d$-dimensional local ring and $I_{1}, I_{2}, \ldots, I_{d}$ be ideals of $R$. Let $R=\left(r_{1}, \ldots, r_{d}\right)$ be a set of nonnegative integers. Denote by $I^{R}$ the ideal $I_{1}^{r_{1}} \cdots I_{d}^{r_{d}}$. Let $R(i)$ denote the set of integers $\left(r_{1}, \ldots, r_{i}-1, \ldots, r_{d}\right)$. Then a set of elements $x_{1}, \ldots, x_{d}$ such that $x_{i} \in I_{i}$ for $i=1,2, \ldots, d$ is called a joint reduction of $I=\left(I_{1}, \ldots, I_{d}\right)$ if for some $R$

$$
I^{R}=x_{1} I^{R(1)}+x_{2} I^{R(2)}+\cdots+x_{d} I^{R(d)} .
$$

THEOREM (REES [R4]). Let $I$ and $J$ be m-primary ideals of a d-dimensional local ring. Then $e_{i}(I \mid J), i=0,1, \ldots, d$ is the multiplicity of the ideal generated by any joint reduction of the set of ideals which consists of $d-i$ copies of $I$ and $i$ copies of $J$.

Since $K=\left(x, y^{2}, z^{2}\right)$ is a reduction of $J=\left(x, y^{2}, y z, z^{2}\right), e_{i}(m \mid J)=e_{i}(m \mid K)$ for all $i$. The equation

$$
m^{2} K^{2}=y m K^{2}+z m K^{2}+x m^{2} K
$$

shows that $(y, z, x)$ is a joint reduction of the set $(m, m, K)$. Therefore $e_{1}(m \mid J)=$ $e(y, z, x)=1$. The equation

$$
m^{2} K^{2}=y m K^{2}+x m^{2} K+z^{2} m^{2} K
$$

shows that $\left(y, x, z^{2}\right)$ is a joint reduction of the set $(m, K, K)$. Therefore $e_{2}(m \mid J)=$ $e\left(y, x, z^{2}\right)=2$. Consequently, $e\left(R[J t]_{N}\right)=e_{0}+e_{1}+e_{2}=1+1+2=4$.

ACKNOWledgment. I would like to thank Professors W. Heinzer and C. Huneke for their help and encouragement. Especially their help in calculating the Hilbert polynomial in $\S 5$ is well appreciated. Thanks are also due to K. N. Raghavan (Purdue University) and Professor R. Sivaramakrishnan (University of Calicut, India) for teaching me how to simplify binomial identities. I want to thank Professor Daniel Katz for listening to my rumblings with great patience. Finally, thanks are due to Professor David Rees for suggesting an improvement in the main theorem over its original version.

\section{REFERENCES}

[B] P. B. Bhattacharya, The Hilbert function of two ideals, Proc. Cambridge Philos. Soc. 53 (1957), 568-575.

[H] C. Huneke, Complete ideals in two dimensional regular local rings (after Zariski and Lipman), Proc. "Microprogram in Commutative Algebra" (MSRI, Berkeley, Calif., 1987) (to appear).

[HS] C. Huneke and J. Sally, Birational extensions in dimension two and integrally closed ideals, J. Algebra 115 (1988), 481-500.

[L] J. Lipman, On complete ideals in regular local rings, preprint, 1987.

[NR] D. G. Northcott and D. Rees, Reductions of ideals in local rings, Proc. Cambridge Philos. Soc. 50 (1954), 145-158.

[R1] D. Rees, $\mathscr{A}$-transforms of local rings and a theorem on multiplicities of ideals, Proc. Cambridge Philos. Soc. 57 (1961), 8-17.

[R2] _ Hilbert functions and pseudo-rational local rings of dimension two, J. London Math. Soc. 24 (1981), 467-479. 
[R3] _ The general extension of a local ring and mixed multiplicities, Algebra, Algebraic Topology and Their Interactions (Proc., Stockholm, 1983), Lecture Notes in Math., vol. 1183, SpringerVerlag, Berlin, 1986, pp. 339-360.

[R4] _ Generalizations of reductions and mixed multiplicities, J. London Math. Soc. 29 (1984), $397-414$.

[Ra] H. Rademacher, Topics in analytic number theory, Springer-Verlag, New York, 1973.

[Ri] J. Riordan, Combinatorial identities, Wiley, New York, 1968.

[T] B. Teissier, Cycles évanescents, sections planes, et conditions de Whitney, Singularitiés à Cargèse, 1972, Astérisque 7-8 (1973), 285-362.

[V] G. Valla, Certain graded algebras are always Cohen-Macaulay, J. Algebra 42 (1976), 537-548.

[Z] O. Zariski, Polynomial ideals defined by infinitely near base points, Amer. J. Math. 60 (1938), 151-204.

[ZS] O. Zariski and P. Samuel, Commutative algebra, Vol. II, Van Nostrand, Princeton, N. J., 1960.

DEPARTMENT OF MATHEMATICS, UNIVERSity OF KANSAS, LAWRENCE, KANSAS 66045 